\title{
ACTIVIDAD FÍSICA Y ATENCIÓN EN ESCOLARES DIAGNOSTICADOS TDAH: REVISIÓN DE ESTUDIOS LONGITUDINALES
}

\section{PHYSICAL ACTIVITY AND ATTENTION IN SCHOOL CHILDREN DIAGNOSED WITH ADHD: REVIEW OF LONGITUDINAL STUDIES}

http://dx.doi.org/10.15304/ie.28.4571

\author{
Sara Suárez-Manzano \\ Universidad de Jaén \\ ssuarez@ujaen.es
}

Alberto Ruiz-Ariza

Universidad de Jaén arariza@ujaen.es

\author{
Sebastián López-Serrano \\ Universidad de Jaén \\ sebalopez0013@gmail.com
}

Emilio J. Martínez López

Universidad de Jaén emilioml@ujaen.es

\section{RESUMEN}

El objetivo de esta revisión fue analizar el efecto de la práctica de Actividad Física (AF) sobre la atención en niños y niñas (6-12 años) con trastorno por déficit de atención e hiperactividad (TDAH). Para ello se hizo una búsqueda bibliográfica en tres bases de datos (búsqueda inicial $n=81$ : PubMed $\mathrm{n}=14$, SportDiscus $\mathrm{n}=16$, Web of Science $\mathrm{n}=51$ ), desde enero de 2000 hasta septiembre de 2017 . Un total de seis estudios longitudinales con intervención cumplieron los criterios de inclusión. Todos los estudios emplearon programas de AF a intensidad moderada y/o vigorosa - $1-5$ sesiones $/ \mathrm{sem}$ con duración de 3-20 semanas. Todos mostraron que la AF mejora la atención en niños y niñas TDAH, además de otras variables como el comportamiento, condición física y motricidad. Finalmente, se observó además relación entre la frecuencia de práctica de AF y el nivel de mejora de la atención. Podemos concluir que los programas de AF, de al menos tres semanas, pueden tener un efecto positivo en la atención de los niños y niñas diagnosticados TDAH. Se necesitan más investigaciones para aclarar el posible papel clave de los factores de confusión.

Palabras clave: Ejercicio Físico, Innovación, Comportamiento, Colegio, Revisión. 


\section{ABSTRACT}

The objective of this review was to analyse the effect of Physical Activity (PA) practice on attention in children (6-12 years) diagnosed with Attention Deficit Hyperactivity Disorder (ADHD). To do this, a bibliographic search was made in three databases (initial search $n=81$ : PubMed $n=14$, SportDiscus $n$ $=16$, Web of Science $\mathrm{n}=51$ ), from January 2000 to September 2017. A total of six longitudinal studies with intervention met the inclusion criteria. All studies used moderate intensity and / or vigorous PA programs - 1-5 sessions / week - with duration of 3-20 weeks. All showed that PA improved attention in ADHD children, in addition to other variables such as behaviour, physical condition and motor skills. Finally, it was also observed a relationship between the frequency of PA practice and the level of improvement of care. We can conclude that PA, programs of at least three weeks, can have a positive effect on the care of children diagnosed with ADHD. More research is needed to clarify the potential key role of confounding factors.

Keywords: Physical Exercise, Innovation, Behaviour, School, Review.

\section{INTRODUCCIÓN}

Con una prevalencia de aproximadamente el 5.3 - 3.4\% entre los jóvenes $\leq 18$ años (Polanczyk, de Lima, Horta, Biederman y Rohde, 2007; Polanczyk, Salum, Sugaya, Caye y Rohde, 2015) el Trastorno por Déficit de Atención y/o Hiperactividad (TDAH) es uno de los trastornos del neurodesarrollo más frecuente en escolares. Estos niños y niñas presentan un patrón persistente de inatención y/o hiperactividad / impulsividad (DSM-5, 2013). Además, manifiestan frecuentemente déficits en las funciones ejecutivas, que comprende, la memoria de trabajo, la atención, el control del comportamiento, la planificación y organización mental (Diamond, 2013). La variable en la que se centra este trabajo es la atención, siendo entendida como el factor responsable de extraer los elementos esenciales para la actividad mental, pudiendo así distinguir principalmente tres aspectos que la constituyen: percepción de forma precisa de objetos y acciones de nuestro entorno, procesamiento rápido de las acciones al recibir un estímulo y mantenimiento de este estado de percepción durante el tiempo que sea necesario para realizar correctamente una acción (Rebollo y Montiel, 2006).

Frecuentemente, los niños y niñas con diagnóstico TDAH reciben tratamientos farmacológicos, basados principalmente en metilfenidato y anfetaminas (Hodgkins, Shaw, Coghill y Hechtman, 2012), estimulantes que elevan los niveles bajos de dopamina en el lóbulo central estriado. Otra estrategia está basada en la modificación de conducta, es decir, ayudarles a controlar la impulsividad y estado emocional, pudiendo de este modo aprovechar las capacidades de percepción (Watson, Richels, Michalek y Raymer, 2015). En jóvenes se ha comprobado que el tratamiento médico es eficaz para mejorar la atención, pero no otras funciones ejecutivas (Biederman et al., 2008). Además, los estudios de Chronis et al. (2004) nos revelan que los efectos beneficiosos de las intervenciones basadas en la modificación de conducta tampoco son efectivos totalmente, ya que los avances durante el tratamiento se disipan rápidamente tras finalizar.

Los bajos niveles de dopamina y norepinefrina están estrechamente correlacionados con los problemas atencionales en niños diagnosticados TDAH (Wolraich et al., 2005). Wigal et al. (2003) observaron que la práctica de ejercicio físico produce la liberación de dopamina en la corteza prefrontal y los ganglios basales. De igual modo, los estudios de Denisco, Tiago y Kravitz (2005) y 
Wolraich et al. (2005) evidencian que la práctica deportiva o ejercicio físico aumenta los niveles de dopamina y norepinefrina en el cerebro.

Sin embargo, en base a los datos revisados, no existe un trabajo que analice los estudios que evalúan el efecto a medio-largo plazo de la práctica de AF debidamente planificada, sobre la atención en niños y niñas (6-12 años) diagnosticados TDAH por especialistas clínicos. La mayoría de los trabajos se centran en conocer el efecto de la AF sobre el comportamiento y funciones ejecutivas de jóvenes (Cerrillo-Urbina et al., 2015; Reeves y Bailey, 2016; Vysniauske et al., 2016).

Este trabajo de revisión está centrado en edad escolar, debido a que la infancia es la etapa idónea para el aprendizaje de hábitos (Capdevilla, Niñerola y Pintanel, 2004). Escogemos población TDAH ante el actual sobrediagnóstico en población española (Isorna Folgar, 2016). Conocer el efecto de diferentes tipos de prácticas físico-deportivas podría ayudar a familiares y docentes a entender cómo pueden mejorar la atención de estos jóvenes sin necesidad exclusiva de recurrir a los medicamentos. Poder ofrecerles una guía para que puedan introducir el movimiento en el día a día de los escolares dentro y fuera del aula, teniendo en cuenta los beneficios que podrían producir no solo a nivel atencional, sino también a su salud metal, social y física, importante objetivo a trabajar en esta etapa desde el contexto familiar escolar y social cercano (Rosales, 2014). En base a lo expuesto, el objetivo fue conocer la influencia que la AF en contexto escolar tiene a medio-largo plazo en los niños y niñas diagnosticados TDAH.

\section{MÉTODO}

El método seguido para este estudio fue de búsqueda bibliográfica. En un primer momento, se determinaron los motores de búsqueda y criterios de selección. Seguidamente cada investigador de este trabajo realizó la búsqueda de manera independiente. Se empleó el gestor bibliográfico EndNote, se pusieron en común los resultados obtenidos para descartar los artículos duplicados y aquellos que no cumplían los límites de búsqueda a nivel de título o resumen (lectura de títulos y lectura de resúmenes). A continuación, individualmente y siguiendo los criterios de selección los investigadores escogieron los artículos que incluirían en el trabajo de revisión (lectura de manuscritos completos). Finalmente, se puso en común la selección y para solucionar las desavenencias entre los investigadores se volvieron a analizar los manuscritos siguiendo los criterios de selección.

\section{Límites de búsqueda}

La búsqueda se realizó en tres bases de datos (PubMed, SportDiscus y Web of Science). Las fechas de búsqueda fueron: enero 2000 - septiembre 2017. Las revistas debían tener revisión por pares y estar indexadas en ISI. Los términos empleados para la búsqueda fueron:

1) Physical activity, physical fitness, cardiovascular fitness, physical education, exercise, physical exercise.

2) Attention-deficit hyperactivity disorder, ADHD, attention deficit disorder, ADD.

3) Children, childhood, school-age youth, student.

4) Attention, executive function, cognition, cognitive performance 


\section{Criterios de selección}

Los estudios seleccionados fueron contrastados con los siguientes criterios:

$1^{\circ}$ Informe completo publicado en una revista revisada por pares.

$2^{\circ}$ La población del estudio fueron escolares diagnosticados TDAH (6 - 12 años).

$3^{\circ}$ El estudio incluyó trabajos escritos en inglés, francés o español.

$4^{\circ}$ El estudio utilizó un diseño con intervención superior a 3 semanas de duración.

$5^{\circ}$ Se midió la variable atención o inatención.

$6^{\circ}$ No existen criterios de exclusión con respecto al origen étnico.

\section{Evidencia del nivel de calidad}

Todos los artículos incluidos en este trabajo son de alta calidad (ver tabla 1). Cumplen los criterios de calidad que se establecieron basados en otras listas de evaluación (Ruiz-Ariza, GraoCruces, de Loureiro y Martínez-López, 2017). De tal modo que se le otorgó cero puntos en el caso de no cumplir la condición propuesta, un punto si se cumplía parcialmente y dos puntos si se cumplía totalmente.

\begin{tabular}{|l|c|c|c|c|c|c|c|c|}
\hline Autores & A & B & C & D & E & F & Puntuación Total & Nivel de calidad \\
\hline Jensen et al. (2004) & 2 & 2 & 2 & 2 & 2 & 2 & 12 & AC \\
McKune et al. $(2004)$ & 1 & 2 & 2 & 2 & 2 & 2 & 11 & AC \\
Verret et al. $(2012)$ & 1 & 2 & 2 & 2 & 2 & 2 & 11 & AC \\
Smith et al. $(2013)$ & 2 & 2 & 2 & 2 & 2 & 2 & 12 & AC \\
Hoza et al $(2015)$ & 2 & 2 & 2 & 2 & 2 & 2 & 12 & AC \\
Meßler et al. (2016) & 2 & 2 & 2 & 2 & 2 & 2 & 12 & AC \\
\hline Leyenda: Alta Calidad (AC)=9-12. \\
Ensayo controlado y aleatorizado. \\
Doble ciego. \\
Revisión por pares. \\
Publicación en idioma inglés, francés o español. \\
Presenta resultados con claridad. \\
Grupo control.
\end{tabular}

Tabla 1. Lista de estudios incluidos con puntuaciones de calidad.

\section{RESULTADOS}

El diagrama de flujo de resultados del proceso de revisión se muestra en la Figura 1. En la búsqueda principal obtuvimos 81 artículos. Se eliminaron siete duplicados, quedando 74 . En el siguiente paso se desestimaron 40, basándonos en la información aportada por el título y resumen. Así, 34 estudios fueron revisados con los criterios de selección, leyendo más a fondo los textos completos. En este último cribado se excluyeron un total de 28 artículos por no cumplir al $100 \%$ 
los criterios de selección. Quedando finalmente seis artículos aptos para ser incluidos en el presente trabajo de revisión (Hoza et al., 2015; Jensen y Kenny, 2004; McKune et al., 2004; Meßler, Holmberg y Sperlich 2016; Smith et al., 2013; Verret et al., 2012).

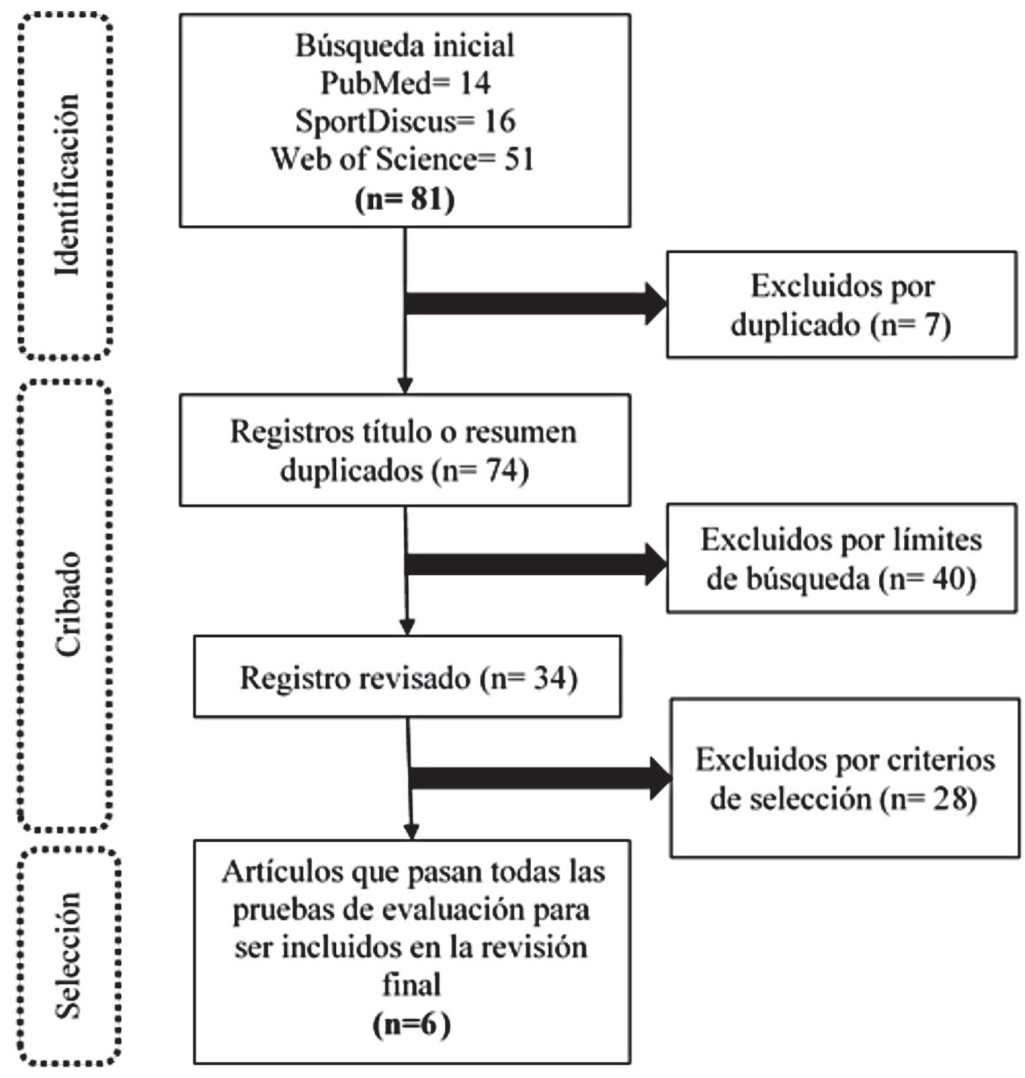

Figura 1. Proceso de selección de artículos.

En este trabajo de revisión se han incluido seis artículos, todos ellos con diseño de intervención, asignando a los participantes de forma aleatoria en los diferentes grupos. Todos ellos evalúan los efectos de un programa de AF en la atención en niños y niñas diagnosticados como TDAH por especialistas psicólogos clínicos (Hoza et al., 2015; Jensen y Kenny, 2004; McKune et al., 2004; Meßler et al., 2016; Smith et al., 2013; Verret et al., 2012).

Esta revisión incluye datos de 297 participantes, siendo el tamaño muestral de los diferentes estudios de entre 13 (McKune et al., 2004) y 202 niños (Hoza et al., 2015). Los estudios se han realizado en cinco países diferentes: dos en USA (Hoza et al., 2015; Smith et al., 2013), uno en Sudáfrica (McKune et al., 2004), uno en Alemania (Meßler et al., 2016), uno en Australia (Jensen y Kenny, 2004) y uno en Canadá (Verret et al., 2012). 


\begin{tabular}{|c|c|c|c|c|c|}
\hline $\begin{array}{l}\text { Autor y } \\
\text { año }\end{array}$ & $\begin{array}{l}\text { Diseño de estudio/ } \\
\text { intervención }\end{array}$ & $\begin{array}{l}\text { Muestra/ } \\
\text { Edad/ } \\
\text { País }\end{array}$ & Grupos & \begin{tabular}{|l|} 
Medidas de \\
Atención, AF y \\
motricidad \\
\end{tabular} & Resultados \\
\hline $\begin{array}{l}\text { Jensen } \text { et al. } \\
\text { (2004) }\end{array}$ & $\begin{array}{l}\text { Intervención/ } \\
\text { Chronic/ } \\
\text { Diagnóstico } \\
\text { DSM-IV/ } \\
20 \text { semanas }\end{array}$ & $\begin{array}{l}19 \text { niños/ } \\
9,99 / \\
\text { Australia }\end{array}$ & $\begin{array}{l}2 \text { grupos: } \\
\text { GE }(\mathrm{n}=11) \text { : yoga } \\
1 \mathrm{~h} / \text { semana } \\
\mathrm{GC}(\mathrm{n}=8) \text { : } \\
\text { no yoga }\end{array}$ & $\begin{array}{l}\text { CPRS. } \\
\text { CTRS. } \\
\text { TOVA. } \\
\text { WISC III } \\
\text { Acelerometría } \\
\text { (Motion Logger } \\
\text { Actigraph) }\end{array}$ & $\begin{array}{l}\text { Los resultados de acelerometría no } \\
\text { fueron concluyentes. } \\
\text { El grupo de yoga mejoró } \\
\text { significativamente la atención } \\
\text { (TOVA y CTRS) y comportamiento } \\
\text { (CPRS y CTRS). } \\
\text { Mejoras significativas para el grupo } \\
\text { de yoga en cinco subescalas de la } \\
\text { CPRS. No habiendo mejoras en GC. }\end{array}$ \\
\hline $\begin{array}{l}\text { McKune et } \\
\text { al. (2004) }\end{array}$ & $\begin{array}{l}\text { Intervención / } \\
\text { Diagnóstico DSM- } \\
\text { III-R. HR/ } \\
5 \text { semanas }\end{array}$ & $\begin{array}{l}13 \text { niños y } \\
\text { niñas/ } \\
11 / \\
\text { South } \\
\text { África }\end{array}$ & $\begin{array}{l}2 \text { grupos: } \\
\text { GE }(\mathrm{n}=8): \mathrm{AF} \\
5 \text { días } / \mathrm{semana} 60 \min (20 \mathrm{~min} \\
50-70 \% \text { of } \mathrm{FCM} \text {, ecuación } \\
\text { Karvonen }) \\
\mathrm{CG}(\mathrm{n}=5) \text { : no ejercicio }\end{array}$ & $\begin{array}{l}\text { CPRS } \\
\text { Mediciones: } 1 \\
\text { semana antes, } 3 \text { y } 5 \\
\text { semanas durante la } \\
\text { intervención }\end{array}$ & $\begin{array}{l}\text { Mejoras significativas en el } \\
\text { comportamiento de ambos grupos } \\
\text { inmediatamente después del } \\
\text { programa: comportamiento total } \\
(p=0,001) \text {, atención }(p=0,008) \text {, } \\
\text { emocional }(p=0,01) \text { y habilidades } \\
\text { motoras }(p=0,004) \text {. }\end{array}$ \\
\hline $\begin{array}{l}\text { Verret et al. } \\
(2012)\end{array}$ & $\begin{array}{l}\text { Intervención/ } \\
\text { Diagnóstico } \\
\text { DSM-IV } \\
10 \text { semanas }\end{array}$ & $\begin{array}{l}21 \text { niños y } \\
\text { niñas/ } \\
9,1 / \\
\text { Canadá }\end{array}$ & $\begin{array}{l}2 \text { grupos: } \\
\text { GE }(\mathrm{n}=10): \mathrm{AF} \\
3 \text { días/semana } 45 \text { minutos } \\
\text { AFMV. } \\
\text { CG }(\mathrm{n}=11) \text { : no ejercicio }\end{array}$ & $\begin{array}{l}\text { El Sky Search } \\
\text { (atención) } \\
\text { TGMD-2 }\end{array}$ & $\begin{array}{l}\text { Los resultados demuestran que } \\
\text { la participación en un programa } \\
\text { de AF mejora la condición física, } \\
\text { comportamiento y nivel de } \\
\text { procesamiento de la información y } \\
\text { atención. }\end{array}$ \\
\hline $\begin{array}{l}\text { Smith et al. } \\
(2013)\end{array}$ & $\begin{array}{l}\text { Intervención/ } \\
\text { Diagnóstico } \\
\text { DSM-V/ } \\
8 \text { semanas }\end{array}$ & $\begin{array}{l}14 \text { niños y } \\
\text { niñas/ } \\
6,7 / \\
\text { USA }\end{array}$ & $\begin{array}{l}1 \text { grupo: } \\
5 \text { días/semana } \\
30 \text { min de AFMV antes de clase }\end{array}$ & \begin{tabular}{|l|} 
WISC-III \\
Bruininks-Oseretsky \\
Test of Motor \\
Proficiency, 2 $2^{\text {a }}$ edición \\
\end{tabular} & $\begin{array}{l}\text { Mejora significativa de la } \\
\text { intervención pre-post observada } \\
\text { en condición física. Gran descenso } \\
\text { de inatención y mejora en el } \\
\text { comportamiento. }\end{array}$ \\
\hline $\begin{array}{l}\text { Hoza et al. } \\
(2015)\end{array}$ & $\begin{array}{l}\text { Intervención/ } \\
\text { Diagnóstico DSM- } \\
\text { IV y ADHD-IV/ } \\
12 \text { semanas }\end{array}$ & $\begin{array}{l}202 \text { niños y } \\
\text { niñas/ } 6,83 / \\
\text { USA }\end{array}$ & $\begin{array}{l}2 \text { grupos: } \\
\text { GE: AF } \\
5 \text { días/semana } 31 \text { min. } \\
\text { GC: } 31 \text { min proyectos artísticos. }\end{array}$ & $\begin{array}{l}\text { Evaluación de } \\
\text { síntomas de } \\
\text { diagnóstico TDAH } \\
\text { PACER }\end{array}$ & $\begin{array}{l}\text { Aumento de la capacidad aeróbica } \\
\text { en GE frente a GC ( } 2,12 \mathrm{Vs} \text {. } 0,74) \text {. } \\
\text { La gravedad de los síntomas en } \\
\text { el grupo de TDAH disminuyó } \\
\text { significativamente en comparación } \\
\text { con GC, para todas las medidas de } \\
\text { gravedad de los síntomas. Redujo la } \\
\text { inatención y el mal humor. }\end{array}$ \\
\hline $\begin{array}{l}\text { Meßler et } \\
\text { al. (2016) }\end{array}$ & $\begin{array}{l}\text { Intervención/ } \\
\text { Diagnóstico/ } \\
\text { ICD } 10 \\
\text { DSM-IV/ } \\
3 \text { semanas }\end{array}$ & $\begin{array}{l}28 \text { niños }(9 \\
\text { TDAH)/ } \\
11 / \\
\text { Alemania }\end{array}$ & $\begin{array}{l}2 \text { grupos: } \\
\text { EG }(\mathrm{n}=5) \text { : HIIT } \\
30 \text { min }(95 \% \text { FCM }) \\
\text { CG }(\mathrm{n}=4): \text { TRAD } 60 \text { min } \\
\text { terapia multimodal estándar } \\
(70 \text { min } \mathrm{AF}<60 \%) \\
3 \text { días/semana }\end{array}$ & $\begin{array}{l}\text { FBB-HKS } \\
\text { SBB-HKS }\end{array}$ & $\begin{array}{l}\text { El GE mejoró autoestima, } \\
\text { socialización y competencia } \\
(\mathrm{p}<0,05) \text {, además de atención. }\end{array}$ \\
\hline \multicolumn{6}{|c|}{$\begin{array}{l}\text { Leyenda: } \mathrm{AF}=\text { Actividad Física. AFMV = Actividad Física de Moderada a Vigorosa intensidad. CPRS = Conners' } \text { Parent Rating Scales. CTRS = } \\
\text { Conners' Teacher Rating Scales. FBB-HKS = cuestionario del trastorno hipercinético evaluado por tutores. FCM = Frecuencia Cardiaca Máxima. } \\
\text { DSM = Diagnostic and Statistical Manual of Mental Disorders. GC = Grupo Control. GE = Grupo Experimental. SBB-HKS = cuestionario } \\
\text { del trastorno hipercinético autoevaluación por los niños. ICD } 10=\text { International Classification of Diseases. PACER = Progressive Aerobic } \\
\text { Cardiovascular Endurance Run. TDAH = Trastorno por Déficit de Atención e Hiperactividad. TOVA = Test Of Variables of Attention. WISC = } \\
\text { Wechsler Intelligence Scale. }\end{array}$} \\
\hline
\end{tabular}

Tabla 2. Seis trabajos de los últimos 17 años que evalúan el efecto de la $\mathrm{AF}$ en la atención en niños y niñas diagnosticados TDAH. 


\section{La atención y su evaluación}

La atención es el factor responsable de extraer los elementos esenciales para la actividad mental. Podemos distinguir dos tipos de atención (Rebollo y Montiel, 2006):

- La atención elemental: también denominada involuntaria, referente al estado generalizado de vigilia o atención. Está vinculada a la vigilia y al estado de alerta de nuestro cuerpo y mente ante posibles estímulos que se puedan percibir en cualquier momento.

- La atención sostenida o la atención selectiva: es la atención referida al estado de activación de los sistemas funcionales, es decir, la que mantiene su activación mientras se incorpora un nuevo estímulo.

En los estudios seleccionados, para evaluar los diferentes aspectos atencionales (atención e inatención) se emplearon diferentes pruebas, CPRS, CTRS, TOVA, WISC III, El Sky Search y los síntomas de inatención del diagnóstico TDAH.

\section{Efecto de la Actividad Física en la atención}

Un total de seis estudios analizaron el efecto crónico de la AF en la atención en niños y niñas diagnosticados TDAH (Hoza et al., 2015; Jensen y Kenny, 2004; McKune et al., 2004; Meßler et al., 2016; Smith et al., 2013; Verret et al., 2012), todos ellos llevaron a cabo una intervención de más de 3 semanas, siendo estudios de carácter longitudinal con intervención. Jensen y Kenny (2004), observaron que los niños con diagnóstico TDAH que asistieron a una sesión de yoga semanal, durante 20 semanas, mejoraban la atención y el comportamiento en el ámbito escolar. Además, detectaron una asociación positiva entre el número de sesiones o número de días de práctica de yoga en casa y el nivel de mejora de la atención. McKune et al. (2004), revelaron que una intervención de AFMV — intensidad 50-70\% FCM —, mejoraba significativamente el comportamiento de los niños y niñas TDAH, a partir de la tercera semana de tratamiento y que, pasadas las cinco semanas de intervención se observaron además mejoras en el nivel control emocional, atencional, y habilidades motrices, frente al grupo control que no manifestó cambios.

Estos resultados son similares a los obtenidos en los otros tres recientes estudios que también emplearon AFMV. Por un lado, Smith et al. (2013) observaron mejoras al comparar las medidas de antes y después de aplicar la intervención, en el comportamiento, inhibición en la respuesta y disminución de la inatención; tras ocho semanas de tratamiento, realizando $30 \mathrm{~min} /$ día al comienzo de cada jornada escolar, cinco días a la semana, de lunes a viernes. Hoza et al. (2015), en su diseño aplicaron en el grupo control una intervención que consistió en realizar proyectos artísticos y el grupo experimental un programa de AF aeróbica. Tras las 12 semanas, en el grupo experimental, registraron mejoras en los síntomas propios del diagnóstico TDAH, tales como en, comportamiento, disminución de la inatención y atenuación del mal humor. Las sesiones se realizaron todos los días de la semana, de lunes a viernes, los $31 \mathrm{~min}$ antes de entrar a clase. Para finalizar, Verret et al. (2012), tras 10 semanas de AFMV, en sesiones de 45 min de duración, realizando únicamente 3 sesiones a la semana, hallaron en el grupo experimental mejora en la condición física y motricidad, además de grandes aumentos en atención, inhibición de respuesta y procesamiento de información. Resultados 
similares a los obtenidos por Meßler et al. (2016) quienes tras tres semanas de intervención empleando método interválico 4 x 4min —intensidad 95\% FCM - $30 \mathrm{~min} /$ día, 3 días/semana, observaron mejoras en atención además de en habilidades motrices y sociabilidad, frente al grupo que participó en sesiones de AF como terapia multimodal estándar — 3 semanas, 3 días/semana, 60 $\min /$ día, intensidad $<70 \% \mathrm{FCM}-$.

\section{DISCUSIÓN}

Este trabajo de revisión ha analizado los estudios que evalúan los efectos de la AF en el ámbito escolar sobre la capacidad de atención o nivel de inatención de los niños (6 - 12 años) diagnosticados TDAH. La búsqueda se limitó a tres bases de datos y a estudios publicados desde enero de 2000 hasta septiembre de 2017. Un total de seis artículos cumplieron los criterios de selección, todos usaron un diseño longitudinal de intervención ( $>$ tres semanas de duración) y evidenciando alta calidad, siendo publicados en algunas de las revistas consideradas como más importantes a nivel mundial. Los programas de AF empleados se basaron en AFMV — entre una y cinco sesiones/sem - con duración de tres a 20 semanas. Los resultados han mostrado que la AF incrementa el nivel de atención y disminuye el nivel de inatención (Hoza et al., 2015; Jensen y Kenny, 2004; McKune et al., 2004; Meßler et al., 2016; Smith et al., 2013; Verret et al., 2012). Además de contribuir positivamente en mejoras del comportamiento (Hoza et al., 2015; Jensen y Kenny, 2004; McKune et al., 2004; Meßler et al., 2016; Smith et al., 2013; Verret et al., 2012), condición física y habilidad motriz (McKune et al., 2004; Meßler et al., 2016; Verret et al., 2012). Ningún estudio reveló asociación negativa.

Existen varios mecanismos para explicar los efectos positivos de la AF sobre la atención. En primer lugar, la correlación existente entre la liberación de catecolamina y la práctica de AF (Muller y Muhlack, 2010; Zouhal, Jacob, Delamarche y Gratas-Delamarche, 2008). En segundo lugar, la práctica de ejercicio físico produce la liberación de dopamina en la corteza prefrontal y los ganglios basales (Muller y Muhlack, 2010, Wigal et al., 2003). En tercer lugar, las concentraciones de adrenalina (epinefrina) y noradrenalina (norepinefrina) aumentan debido a la práctica de ejercicio físico (Zouhal et al., 2008). Además, mejora la condición física, variable que se asocia positivamente con el rendimiento cognitivo (Ardoy, Fernández-Rodríguez, Jiménez-Pavón, Castillo, Ruiz y Ortega 2014) y el rendimiento académico en matemáticas y lenguaje (Carral, Pérez y Espiño, 2016). Finalmente, los niveles de dopamina y norepinefrina están estrechamente correlacionadas con la atención y los síntomas cognitivos del TDAH (Wolraich et al., 2005). Por lo cual, el movimiento e intensidad que incrementan el metabolismo basal de los niños pudieran ser la causa de los cambios fisiológicos (catecolamina, dopamina y norepinefrina) que a su vez afectan directamente al aumento de atención y disminución de inatención (Denisco, Tiago y Kravitz, 2005).

A todo esto se suma la mejora que produce la AF de la condición física y habilidades motrices (Hardman y Stensel, 2009), que se asocian positivamente con la mejora de las funciones cognitivas (Gapin y Etnier, 2010). Debemos tener en cuenta que Jensen y Kenny (2004), consideraron la posible influencia del número de sesiones y número de días de práctica de yoga, sobre los beneficios que aporta el programa de AF. Obtuvieron efecto directo de ambas variables sobre el nivel de 
impulsividad, inatención y comportamiento de los niños diagnosticados TDAH tras finalizar la intervención. En los demás estudios no se han estudiado efectos de las posibles variables confusoras que pudieran influir en los efectos del programa de AF.

Sin embargo, si hacemos una rápida búsqueda en las mismas bases de datos, ampliando los términos a niños que no sean diagnosticados con ningún tipo de trastorno neurocognitivo, nos encontramos con una gran variedad de estudios que emplean diversas covariables, tales como la educación maternal (Esteban-Cornejo et al., 2014), indicadores de condición física e índice de masa corporal (Sardinha, Marques, Martins, Palmeira y Minderico, 2014), porcentaje de grasa corporal (Cadenas-Sánchez et al., 2016), o tiempo de lactancia materna (Luby, Belden, Whalen, Harms y Barch, 2016).

Este trabajo de revisión presenta varias limitaciones que debemos reconocer. Limitaciones tales como el pequeño número de artículos incluidos finalmente, a pesar de que su homogeneidad se optimizó mediante un riguroso proceso de selección basado en criterios de selección y exclusión. La mayoría de las investigaciones analizan un número pequeño de individuos, debido quizás a que la prevalencia del TDAH es de aproximadamente el 5\% de la población juvenil (Polanczyk et al., 2007). La mayoría de los escolares que participaron en los estudios son niños y no niñas, debido tal vez a una mayor prevalencia del TDAH en chicos que en chicas $(9,2 \%$ versus $3 \%)$. En algunos casos no se especifica si los participantes interrumpieron o modificaron su tratamiento farmacológico durante el estudio o en las horas previas a la medición, factor que puede alterar en gran medida los resultados de los test de atención. Además, no tienen en cuenta la AF adicional que podrían llevar a cabo los participantes en su vida cotidiana, los desplazamientos activos a la escuela, actividades extraescolares como deportes federados, escuelas multideporte o clases de danza, o inclusive la actividad durante la jornada escolar (descansos entre clases y recreo).

Por otra parte, este trabajo de revisión tiene grandes fortalezas. Por un lado, todos los estudios seleccionados incluyen escolares diagnosticados por especialistas que han empleado para el diagnóstico clínico pruebas y baremos estandarizados y validados, descartando otro tipo de problemas mentales o psicóticos. Por otro lado, todos los trabajos describen claramente la variable dependiente (atención) y la variable independiente (el programa de intervención) concretando minuciosamente el tipo de AF que realizan, la frecuencia, y en la mayor parte de los casos, incluso la intensidad a la que se realiza. Además, este trabajo abarca investigaciones llevadas a cabo en cinco países diferentes, en un periodo temporal de 17 años, en revistas de lengua inglesa, con revisión por pares y siendo todos los artículos incluidos de gran calidad. Por último, esta revisión incluyó solo estudios de carácter longitudinal con intervención superior a tres semanas de programa de AF y llevado a cabo por especialistas desde el ámbito escolar.

Basándonos en los resultados de los diferentes artículos, todas las intervenciones tuvieron una duración alrededor de entre tres y 20 semanas, con una frecuencia de uno, tres o cinco días por semana de ejercicio físico. La duración de las sesiones oscila entre 30 y 60 min. La Organización Mundial de la Salud (OMS, 2010), recomienda que los escolares de cinco a 17 años inviertan como mínimo 60 min diarios en actividades físicas de intensidad moderada a vigorosa. Actividades en las que se comprenden juegos, deportes, desplazamientos, actividades recreativas, educación física 
o ejercicios programados, en el contexto de la familia, la escuela o las actividades comunitarias. Con el fin de mejorar las funciones cardiorrespiratorias, musculares y óseas, así como reducir el riesgo de padecer enfermedades no transmisibles. Sin embargo, ninguno de estos estudios nos indica la AF total que realizan estos jóvenes, no pudiendo confirmar si cumplen o no las recomendaciones de la OMS, al igual que no informan de otras posibles variables confusoras que podrían influir en gran medida en los resultados obtenidos tras la aplicación de una intervención basada en AF.

\section{CONCLUSIONES}

La presente revisión seleccionó un total de seis artículos de intervención, que analizaron el efecto de un programa de AF de al menos tres semanas de duración sobre la variable atencional, en escolares de entre seis y 12 años, diagnosticados TDAH por especialistas. Todos los estudios analizados $(100 \%)$ han mostrado efectos beneficiosos de la $\mathrm{AF}$ en la atención o inatención de jóvenes TDAH, además de otros beneficios a nivel comportamental y condición física. El ejercicio a una intensidad moderada-vigorosa y especialmente aquellos que trabajan la resistencia cardiorrespiratoria, han mostrado una mayor mejora en la atención en estudiantes de primaria TDAH. Estudios recientes evidencian que los jóvenes presentan un alto abandono de práctica de AF y deportiva (Isorna Folgar, Ruiz Juan y Rial Boubeta, 2013), siendo los principales predictores de la inactividad el género, el tiempo de pantalla, el entorno familiar y el entorno o amistades, aparecieron como fuertes predictores del estilo de vida de los jóvenes (Vidal-Conti, 2016). Hemos observado que los beneficios se amplían en función del tiempo de intervención. Además, la AF mejora no solo la atención, sino también la inhibición, control de las emociones, comportamiento, condición física, motricidad y por supuesto la salud. Al realizar un programa de AF debidamente estructurado, de al menos tres semanas de duración. Se puede practicar multitud de deportes y actividades físicas, muchas de ellas fácilmente adaptables a la capacidad, motivación, y disponibilidad de recursos humanos, materiales y temporales. Deportes como la natación, el tenis de mesa o la esgrima, son ideales para disminuir la inquietud y mejorar la atención (Reche García, 2016). Queremos destacar la posibilidad de emplear videojuegos activos y demás dispositivos electrónicos, con el fin de motivar al movimiento gracias al aliciente que aportan las TIC (Beltrán Carrillo, Beltrán Carrillo, Moreno Murcia, Cervelló Gimeno y Montero Carretero, 2012).

No obstante, más investigaciones son necesarias para justificar el efecto diferencial a corto, medio y largo plazo de la AF sobre la atención en jóvenes con dificultades de aprendizaje. La utilización de programas que combinen AF y control conductual deberían ser promovidos durante la infancia desde el ámbito educativo y familiar. Especialmente, se recomienda seguir estudiando en esta línea para clarificar la influencia de variables confusoras, para así poder precisar el tipo de AF y el momento del día ideales para poder aprovechar de forma más eficiente los beneficios que el movimiento puede aportar. 


\section{REFERENCIAS BIBLIOGRÁFICAS}

American Psychiatric Association (2013). Diagnostic and Statistical Manual of Mental Disorders (DSM-5®). American Psychiatric Association.

Ardoy, D. N., Fernández-Rodríguez, J. M., Jiménez-Pavón, D., Castillo, R., Ruiz, J. R. y Ortega, F. B. (2014). A Physical Education trial improves adolescents' cognitive performance and academic achievement: the EDUFIT study. Scandinavian journal of medicine \& science in sports, 24(1), pp. 52-61. DOI: http://dx.doi.org/10.1111/sms.12093

Beltrán Carrillo, V. J., Beltrán Carrillo, J. I., Moreno Murcia, J. A., Cervelló Gimeno, E. y Montero Carretero, C. (2012). El uso de videojuegos activos entre los adolescentes. Cultura, Ciencia y Deporte, 7(19), pp. 19-24. DOI: http://dx.doi.org/10.12800/ccd.v7i19.20

Biederman, J., Seidman, L. J., Petty, C. R., Fried, R., Doyle, A. E., Cohen, D. R., ... y Faraone, S. V. (2008). Effects of stimulant medication on neuropsychological functioning in young adults with attention-deficit/hyperactivity disorder. Journal of Clinical Psychiatry, 69(7), pp. 11501156. DOI: https://doi.org/10.4088/JCP.v69n0715

Cadenas-Sánchez, C., Mora-González, J., Migueles, J.H., Martín-Matillas, M., Gómez-Vida, J., Escolano-Margarit, M.V., ... y de Teresa, C. (2016). An exercise-based randomized controlled trial on brain, cognition, physical health and mental health in overweight/obese children (ActiveBrains project): Rationale, design and methods. Contemporary Clinical Trials, 47, pp. 315-324. DOI: http://dx.doi.org/10.1016/j.cct.2016.02.007

Capdevila, L., Niñerola, J. y Pintanel, M. (2004). Motivación y actividad física: el autoinforme de motivos para la práctica de ejercicio físico (AMPEF). Revista de Psicología del Deporte, 13(1), pp. 55-74.

Carral, J. M. C., Pérez, C. A., y Espiño, M. J. S. (2016). Relación entre la condición física y rendimiento académico en matemáticas y lenguaje en estudiantes españoles de educación secundaria: Un estudio longitudinal. Cultura, Ciencia y Deporte, 11(31), pp. 7-16. DOI: http:// dx.doi.org/10.12800/ccd.v11i31.638

Cerrillo-Urbina, A. J., García-Hermoso, A., Sánchez-López, M., Pardo-Guijarro, M. J., Santos Gómez, J. L., y Martínez-Vizcaíno, V. (2015). The effects of physical exercise in children with attention deficit hyperactivity disorder: a systematic review and meta-analysis of randomized control trials. Child: care, health and development, 41(6), pp. 779-788. DOI: http://dx.doi. org/10.1111/cch.12255

Chronis, A. M., Fabiano, G. A., Gnagy, E. M., Onyango, A. N., Pelham, W. E., Lopez-Williams, A., ... y Seymour, K. E. (2004). An evaluation of the summer treatment program for children with attention-deficit/hyperactivity disorder using a treatment withdrawal design. Behavior Therapy, 35(3), pp. 561-585. DOI: http://dx.doi.org/10.1016/S0005-7894(04)80032-7

Denisco, S., Tiago, C. y Kravitz, C. (2005). Evaluation and treatment of pediatric ADHD. The Nurse Practitioner, 30(8), pp. 14-23. DOI: http://dx.doi.org/10.1097/00006205-200508000-00004

Diamond, A. (2013). Executive functions. Annual review of psychology, 64, pp. 135. DOI: http:// dx.doi.org/10.1146/annurev-psych-113011-143750

Esteban-Cornejo, I., Tejero-González, C. M., Mártinez-Gómez, D., del-Campo, J., González-Galo, A., Padilla-Moledo, C., ... y group, D. study. (2014). Independent and combined influence of 
the components of physical fitness on academic performance in youth. The Journal of pediatrics, 165(2), pp. 306-312. DOI: http://dx.doi.org/10.1016/j.jpeds.2014.04.044

Gapin, J. y Etnier, J. L. (2010). The Relationship Between Physical Activity and Executive Function Performance in Children with Attention-Deficit Hyperactivity Disorder. Journal of Sport \& Exercise Psychology, 32(6), pp. 753-763. DOI: https://doi.org/10.1123/jsep.32.6.753

Hardman, A. E. y Stensel, D. J. (2009). Physical activity and health: the evidence explained. Routledge. DOI: https://doi.org/10.4324/9780203890714

Hodgkins, P., Shaw, M., Coghill, D. y Hechtman, L. (2012). Amfetamine and methylphenidate medications for attention-deficit/hyperactivity disorder: complementary treatment options. European child \& adolescent psychiatry, 21(9), pp. 477-492. DOI: http://dx.doi.org/10.1007/ s00787-012-0286-5

Hoza, B, Smith, A. L., Shoulberg, E. K., Linnea, K. S., Dorsch, T. E., Blazo, J. A., ... y McCabe, G. P. (2015). A Randomized Trial Examining the Effects of Aerobic Physical Activity on Attention-Deficit/Hyperactivity Disorder Symptoms in Young Children. Journal of Abnormal Child Psychology, 43(4), pp. 655-667. DOI: http://dx.doi.org/10.1007/s10802-014-9929-y

Isorna Folgar, M. (2016). El niño hiperactivo y las polémicas en torno al diagnóstico y al tratamiento del TDAH. Innovación educativa, 26, pp. 181-200. DOI: http://dx.doi.org/10.15304/ ie. 26.2913

Isorna Folgar, M., Ruiz Juan, F. y Rial Boubeta, A. (2013). Variables predictoras del abandono de la práctica físico-deportiva en adolescentes. Cultura, Ciencia y deporte, 8(23), pp. 93-102. DOI: http://dx.doi.org/10.12800/ccd.v8i23.297

Jensen, P. S., y Kenny, D. T. (2004). The effects of yoga on the attention and behavior of boys with attention-deficit/hyperactivity disorder (ADHD). Journal of Attention Disorders, 7(4), pp. 205-216. DOI: http://dx.doi.org/10.1177/108705470400700403

Luby, J. L., Belden, A. C., Whalen, D., Harms, M. P. y Barch, D. M. (2016). Breastfeeding and Childhood IQ: The Mediating Role of Gray Matter Volume. Journal of the American Academy of Child \& Adolescent Psychiatry. 55(5), pp. 367-375. DOI: http://dx.doi.org/10.1016/j. jaac.2016.02.009

McKune, A. J., Pautz, J. y Lomjbard, J. (2004). Behavioural response to exercise in children with attention-deficit/hyperactivity disorder. South African Journal of Sports Medicine, 15(3), pp. 17-21. DOI: http://dx.doi.org/10.17159/2413-3108/2003/v15i3a223

Meßler, C. F., Holmberg, H. C. y Sperlich, B. (2016). Multimodal therapy involving high-intensity interval training improves the physical fitness, motor skills, social behavior, and quality of life of boys with ADHD: A randomized controlled study. Journal of attention disorders, pp. 1-7. DOI: http://dx.doi.org/10.1177/1087054716636936

Müller, T. y Muhlack, S. (2010). Effect of exercise on reactivity and motor behaviour in patients with Parkinson's disease. Journal of Neurology, Neurosurgery \& Psychiatry, 81(7), 747-753. DOI: http://dx.doi.org/10.1136/jnnp.2009.174987

OMS-Organización Mundial de la Salud (2010). Recomendaciones mundiales sobre actividad física para la salud. Ginebra, Suiza.

Polanczyk, G., de Lima, M. S., Horta, B. L., Biederman, J. y Rohde, L. A. (2007). The worldwide prevalence of ADHD: a systematic review and metaregression analysis. The American journal of psychiatry, 164(6), pp. 942-948. DOI: http://dx.doi.org/10.1176/ajp.2007.164.6.942 
Polanczyk, G. V, Salum, G. A., Sugaya, L. S., Caye, A. y Rohde, L. A. (2015). Annual Research Review: A meta-analysis of the worldwide prevalence of mental disorders in children and adolescents. Journal of Child Psychology and Psychiatry, 56(3), pp. 345-365. DOI: http:// dx.doi.org/10.1111/jcpp.12381

Rebollo, M. A. y Montiel, S. (2006). Atención y funciones ejecutivas. Revista de neurología, 42(2), pp. 3-7.

Reche García, C. (2016). La esgrima extraescolar como propuesta didáctica en TDAH. Cultura, Ciencia y Deporte, 11(33), pp. 217-224. DOI: https://dx.doi.org/10.12800/ccd.v11i33.766

Reeves, M. J. y Bailey, R. P. (2016). The effects of physical activity on children diagnosed with attention deficit hyperactivity disorder: a review. Education 3-13, 44(6), 591-603. DOI: https:// doi.org/10.1080/03004279.2014.918160

Rosales, C. (2014). Salud y estilos de vida de alumnos de educación primaria. Innovación educativa, 24, pp. 289-303. DOI: http://dx.doi.org/10.15304/ie.24.2047

Ruiz-Ariza, A., Grao-Cruces, A., de Loureiro, N. E. M. y Martínez-López, E. J. (2017). Influence of physical fitness on cognitive and academic performance in adolescents: A systematic review from 2005-2015. International Review of Sport and Exercise Psychology, 10(1), pp. 108-133. DOI: http://dx.doi.org/10.1016/j.gaceta.2015.06.002

Sardinha, L. B., Marques, A., Martins, S., Palmeira, A. y Minderico, C. (2014). Fitness, fatness, and academic performance in seventh-grade elementary school students. BMC pediatrics, 14(1), pp. 176. DOI: http://dx.doi.org/10.1186/1471-2431-14-176

Smith, A. L., Hoza, B., Linnea, K., McQuade, J. D., Tomb, M., Vaughn, A. J., .. y Hook, H. (2013). Pilot Physical Activity Intervention Reduces Severity of ADHD Symptoms in Young Children. Journal of Attention Disorders, 17(1), pp. 70-82. DOI: http://dx.doi. org/10.1177/1087054711417395

Vaidya, C. J., Austin, G., Kirkorian, G., Ridlehuber, H. W., Desmond, J. E., Glover, G. H. y Gabrieli, J. D. E. (1998). Selective effects of methylphenidate in attention deficit hyperactivity disorder: a functional magnetic resonance study. Proceedings of the National Academy of Sciences, 95(24), pp. 14494-14499. DOI: http://dx.doi.org/10.1073/pnas.95.24.14494

Verret, C., Guay, M. C., Berthiaume, C., Gardiner, P. y Beliveau, L. (2012). A Physical Activity Program Improves Behavior and Cognitive Functions in Children With ADHD: An Exploratory Study. Journal of Attention Disorders, 16(1), pp. 71-80. DOI: http://dx.doi. org/10.1177/1087054710379735

Vidal-Conti, J. (2016). Identificación de predictores de actividad física en escolares según el modelo socio-ecológico mediante un análisis multifactorial. Cultura, Ciencia y Deporte, 11(31), pp. 51-59. DOI: http://dx.doi.org/10.12800/ccd.v11i31.642

Vysniauske, R., Verburgh, L., Oosterlaan, J. y Molendijk, M. L. (2016). The effects of physical exercise on functional outcomes in the treatment of ADHD: a meta-analysis. Journal of attention disorders, 1087054715627489. DOI: https://doi.org/10.1177/1087054715627489

Watson, S. M. R., Richels, C., Michalek, A. P. y Raymer, A. (2015). Psychosocial Treatments for ADHD A Systematic Appraisal of the Evidence. Journal of Attention Disorders, 19(1), pp. 3-10. DOI: http://dx.doi.org/10.1177/1087054712447857 
Wigal, S. B., Nemet, D., Swanson, J. M., Regino, R., Trampush, J., Ziegler, M. G. y Cooper, D. M. (2003). Catecholamine response to exercise in children with attention deficit hyperactivity disorder. Pediatric research, 53(5), pp. 756-761. DOI: http://dx.doi.org/10.1203/01. PDR.0000061750.71168.23

Wolraich, M. L., Wibbelsman, C. J., Brown, T. E., Evans, S. W., Gotlieb, E. M., Knight, J. R., ... y Wilens, T. (2005). Attention-deficit/hyperactivity disorder among adolescents: a review of the diagnosis, treatment, and clinical implications. Pediatrics, 115(6), pp. 1734-1746. DOI: http://dx.doi.org/10.1542/peds.2004-1959

Zouhal, H., Jacob, C., Delamarche, P. y Gratas-Delamarche, A. (2008). Catecholamines and the effects of exercise, training and gender. Sports Medicine, 38(5), pp. 401-423. DOI: http:// dx.doi.org/10.2165/00007256-200838050-00004 